\title{
Just On Time - A Concept for iPad Enabled Timely Accurate Continuous Descent Operations
}

\author{
Helge Lenz, Ralf Kohrs \\ German Aerospace Center DLR, Braunschweig, Germany
}

\begin{abstract}
Continuous Descent Operations have the capability to lower noise emissions as well as fuel consumption. Due to their limited flexibility in terms of speed and lateral route variations during the descent phase they are only used in low density traffic situations. Speed commands and radar vectors are used nowadays to build an optimal arrival sequence in medium to high traffic density situations. With the transition to trajectory based operations in the terminal area this limitation could be overcome by time based separation and assigning required times of arrival (RTAs) to the approaching aircraft at a metering fix close to the runway. However, only a limited number of aircraft are equipped with a flight management system (FMS) capable of meeting an RTA in a descent phase with the required accuracy.
\end{abstract}

Within the Clean Sky WP3 a concept named Time and Energy Managed Operations (TEMO) has been developed. It uses optimization technology to re-calculate an energy optimal descent trajectory during flight. Energy errors are compensated by modifying the speed and altitude profile of the approach and if necessary thrust or speed brakes are added to meet a required time of arrival. To fly TEMO advanced FMS and flight deck functions are needed such as direct autopilot access to the speed brakes or the thrust system. As none of these functions are available on board of todays' transport type aircraft DLR proposed a concept enabling the flight crew to fly 4D operations including TEMO with a minimum of additional hardware. The pilot sets the target speed and altitude of the autopilot according to the guidance information provided on an Electronic Flight Bag (EFB) so that the aircraft follows the descent profile in space and in time. The proposed target speeds are calculated by a controller to minimize the time error which also indicates the use of speed brakes or autopilot V/S mode if the altitude error exceeds a predefined threshold.

This paper describes the development and verification of an iPad based 4D guidance display that enables to fly highly accurate 4D trajectories with transport aircraft in the market today. Following steps are included:

- Concept development

- HMl design and implementation

- Simulation campaign investigating operator acceptance

- Flight test preparation

- Flight test campaign to demonstrate feasibility and overall performance

\section{Concept development}

To avoid certification issues and enable to fly 4D CDO also with aircraft on the market today the guidance algorithm has no direct connection to the aircraft systems. An independent guidance system running on an Electronic Flight Bag (e.g. iPad) calculates the necessary speed and altitude commands to follow a pre-calculated 4D trajectory. The pilot closes the control loop by manual input of speed and altitude target values to the autopilot. Any tactical errors regarding arrival time or altitude are directly compensated by the 4D controller and displayed on the EFB.

\section{HMI design and implementation}

To display the target values generated by the 4D controller to the pilot an HMI on an Apple iPad has been developed (see Figure 1). With an appropriate interface the iPad can be certified as class 2 EFB device in the cockpit and used during all phases of flight. 


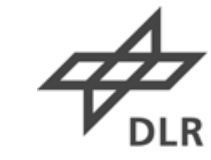

Deutsches Zentrum

DLR für Luft- und Raumfahrt

German Aerospace Center

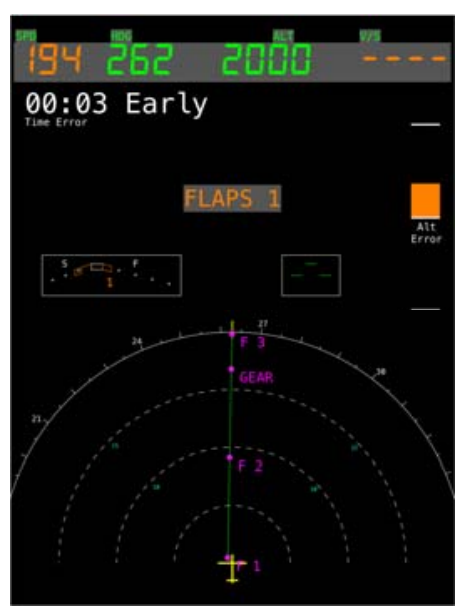

Figure 1: 4D guidance display on the iPad

\section{Simulation campaign investigating operator acceptance}

In 2014 a first simulator campaign (see Figure 2) with four airline pilots has been conducted. In a structured interview feedback regarding the overall feasibility of the operation, the particular HMI implementation and the subjective workload at different update rates were gathered. Additionally simulator performance data was recorded including time accuracy at the final approach fix and pilot reaction time to speed or altitude commands.

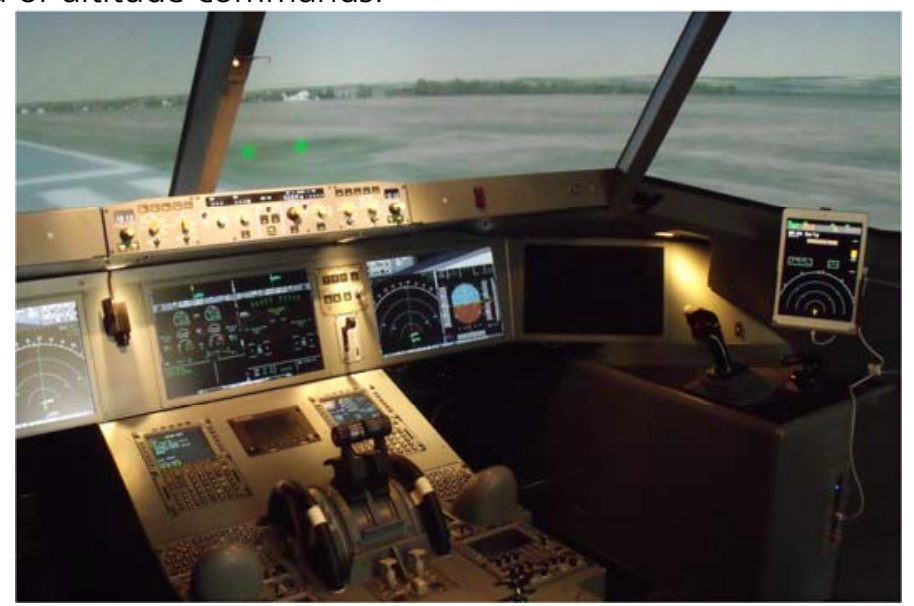

Figure 2: iPad mounted in the simulator

\section{Flight test preparation}

Following the successful simulator campaign the preparation for a flight test started with the improvement of the 4D guidance module to fit to the particular test aircraft: DLRs Advanced Technology Research Aircraft (ATRA), an Airbus A320-232 equipped with flight test installations to access and record over 230 parameter in flight. As the certified aircraft interface module for the iPad was not available for the flight trials the preparation also included a certification and installation of a wired network interface between the iPad and the experimental on-board local area network (see Figure 3). 


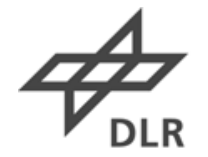

Deutsches Zentrum

für Luft- und Raumfahrt

German Aerospace Center

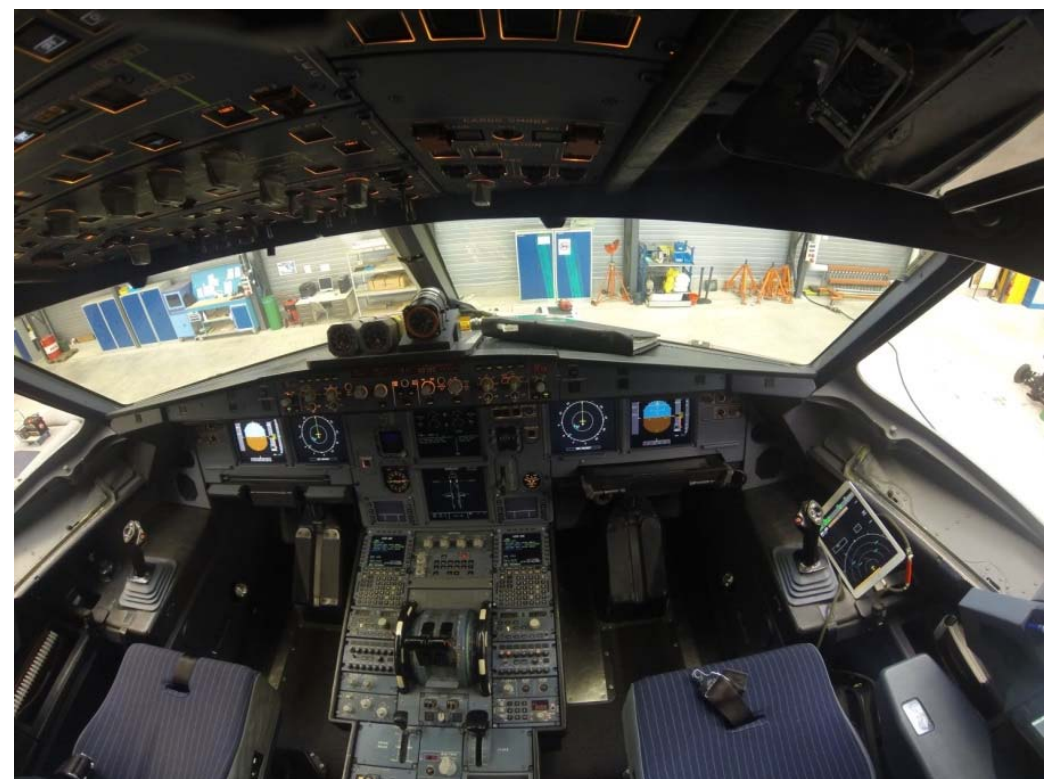

Figure 3: iPad installed in the A320 cockpit (stowed next to right side stick during test preparation)

\section{Flight test campaign to demonstrate feasibility and overall performance}

The flight test campaign started in November 2015 with four approaches into BraunschweigWolfsburg Airport from FL100 and FL240 at different weather conditions. Already during these approaches time errors of less than 10s from top of descent down to the glideslope intercept could be recorded. However due to inaccuracies in the wind forecast and the trajectory generator module the altitude error exceeded $1000 \mathrm{ft}$ during the approaches. To compensate this error the 4D guidance module commanded a vertical speed of less than the predicted vertical speed to compensate the error and guide the aircraft back on the predicted vertical profile.

To improve the performance model inside the trajectory generator module flight test recordings of recent continuous descent approaches were used and extensively tested. After this another three approaches from FL240 were conducted in March 2016 which showed only minor altitude errors during the approach while the time accuracy could be even improved.
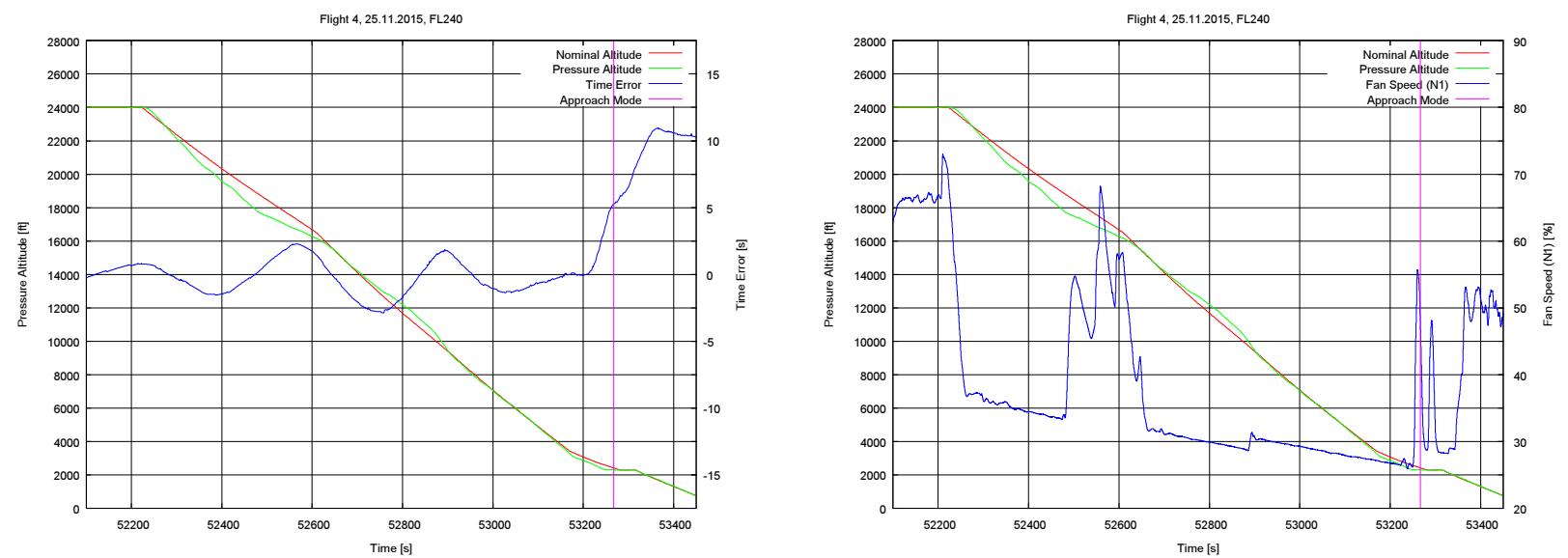

Figure 4: Altitude and Time Error / Fan Speed N1 before trajectory model improvements As shown in Figure 4 an altitude error leads to a vertical speed command around FL180 while after the improvements of the trajectory generator module thrust was only needed due to operational level offs in FL100 and at $4000 \mathrm{ft}$ (see Figure 5). 

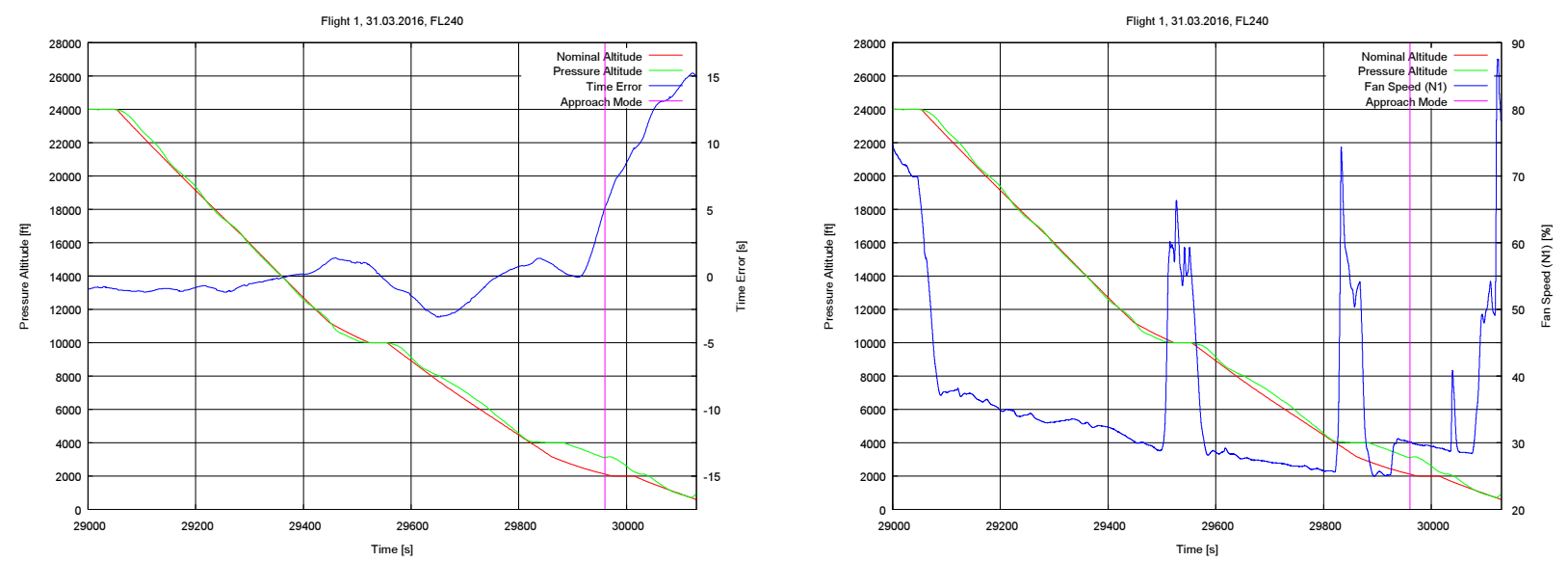

Figure 5: Altitude and Time Error / Fan Speed N1 after trajectory model improvements

\section{References}

[1] Lenz, H., "DLR-ATRA Flight Test - CleanSky Test Flights", CleanSky SGO-ITD O_3.3.2_16, Version A0.2, January 2016

[2] Witt, T., "Development and evaluation of an EFB based display to assist pilots flying environmatally optimized 4D approach procedures", Diploma Thesis, March 2015 